\title{
A paramutation phenomenon is involved in the genetics of maize low phytic acid1-241 (Ipa1-241) trait
}

\author{
R Pilu ${ }^{1,4}$, D Panzeri ${ }^{1,4}$,E Cassani ${ }^{1}$, F Cerino Badone ${ }^{1}$, M Landoni ${ }^{2}$ and E Nielsen ${ }^{3}$ \\ ${ }^{1}$ Dipartimento di Produzione Vegetale, Università degli Studi di Milano, Milano, Italy; ${ }^{2}$ Dipartimento di Scienze Biomolecolari \\ e Biotecnologie, Università degli Studi di Milano, Milano, Italy and ${ }^{3}$ Dipartimento di Genetica e Microbiologia, Università degli Studi di \\ Pavia, Pavia, Italy
}

\begin{abstract}
So far, in maize, three classes of mutants involved in phytic acid biosynthesis have been isolated: Ipa1, Ipa2 and Ipa3. In 2007, a gene tagging experiment performed by Shi et al. found that mutations in ZmMRP4 (multidrug resistance-associated proteins 4) gene cause Ipa1 phenotype. In previous studies, we isolated and described a single recessive Ipa mutation (originally named Ipa241), which was allelic to the Ipa1-1 mutant, and was consequently renamed Ipa1-241. It showed a decrease in the expression of the myo-inositol (Ins)-3phosphate synthase gene (mips1S). In this study, we present genetic and molecular analyses of the Ipa1-241 mutation that
\end{abstract}

indicate an epigenetic origin of this trait, that is, a paramutagenic interaction that results in meiotically heritable changes in ZmMRP4 gene expression, causing a strong pleiotropic effect on the whole plant. The use of a 5-Azacytidine treatment provided data suggesting an association between gene methylation and the Ipa1-241 phenotype. To our knowledge, this is the first report of a paramutagenic activity not involving flavonoid biosynthesis in maize, but regarding a key enzyme of an important metabolic pathway in plants.

Heredity (2009) 102, 236-245; doi:10.1038/hdy.2008.96; published online 10 September 2008

Keywords: maize; phytic acid; 5-Azacytidine treatment; gene silencing; paramutation

\section{Introduction}

Phytic acid (myo-inositol (Ins)-1,2,3,4,5,6-hexakisphospate, or InsP6) is the major phosphorus storage form in cereal seeds as well as in plants in general ( $\mathrm{O}^{\prime}$ Dell et al., 1972; Raboy, 1990). The biosynthetic route begins with Ins and can be summarized as showed in Figure 1. Myo-Ins, in addition to being phosphorylated to hexakis phosphate (phytic acid) during seed maturation, plays a central role in several metabolic processes and in signal transduction in the plant cell (Johnson and Wang, 1996; Raychaudhuri and Majumder 1996; Majumder et al., 1997; Raychaudhuri et al., 1997). Thus, the free myo-Ins level may influence plant growth, development and responses to variations of environmental conditions (Munnik et al., 1998; Stevenson et al., 2000).

In maize, three different recessive low phytic acid (lpa) mutants have so far been isolated (lpa1, lpa2 and lpa3) and involved in this pathway (Figure 1). They produce seeds with reduced phytic acid content and a proportionally higher level of free phosphate, whereas the total amount of seed P is not significantly altered (Raboy et al., 2000, Pilu et al., 2003; Shi et al., 2005). The high inorganic phosphate phenotype (HIP) associated with the lpal

Correspondence: $\operatorname{Dr} R$ Pilu, Dipartimento di Produzione Vegetale, University of Milano, Via celoria 2, Milano 20133, Italy.

E-mail: salvatore.pilu@unimi.it

${ }^{4}$ These authors contributed equally to this work.

Received 26 March 2008; revised 1 July 2008; accepted 8 August 2008; published online 10 September 2008 phenotype can be quickly determined using Chen's assay (Chen et al., 1956), so lpa mutant seeds can easily be screened. Regarding lpa1 mutants, there is evidence indicating that they map on the short arm of chromosome 1, where a mips1s sequence has been localized too (Raboy et al., 2000).

Although biochemical, mapping and gene expression data suggest the mips1s gene as a candidate for lpa1 mutation in maize (Raboy et al., 2000; Pilu et al., 2003; Shukla et al., 2004; Pilu et al., 2005), recent transposon mutagenesis experiments found that a novel gene designated ZmMRP4 (accession number EF86878), coding a multidrug resistance-associated-protein (MRP) mapping near the mips1s sequence, is the actual responsible gene for lpa1 mutation (Shi et al, 2007). MRP proteins represent a subfamily of ATP-binding cassette transmembrane transporters widespread in all eukaryotes, which in plants are involved in several functions, such as xenobiotic detoxification, organic ions transport, oxidative stress tolerance (Swarbreck et al., 2003) and even transpiration control (Klein et al., 2006).

We have previously isolated a single recessive lpa mutation in maize, named lpa241, which appeared to be allelic to lpa1-1, and was therefore renamed lpa1-241. As independently found for both $1 p a 1-1$ and lpa1-241 alleles, mips1S gene expression was reduced in developing seeds (Pilu et al., 2003; Shukla et al., 2004), but in both alleles no molecular lesions in the gene coding region were found (Shukla et al., 2004; Pilu et al., 2005).

Moreover, lpa1-241 plants exhibit a variety of morphological and physiological alterations related to the lpa 


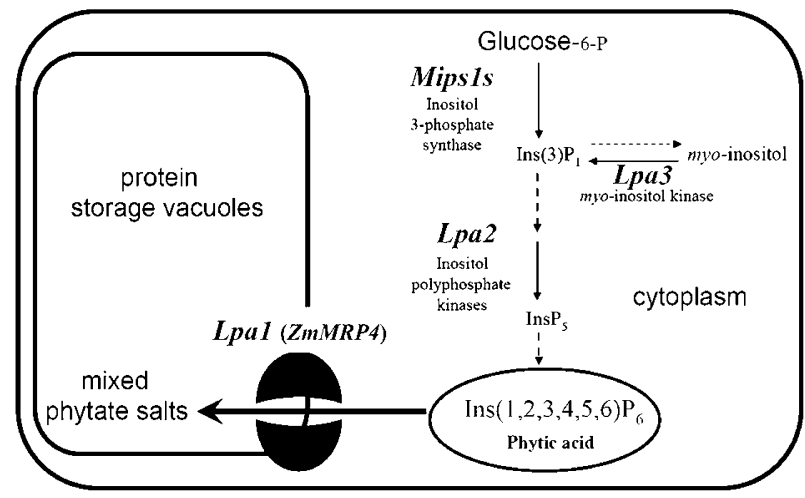

Figure 1 Schematic model of the biosynthetic pathways leading to phytic acid accumulation in protein storage vacuoles of the maize seed. Main known genes involved in inositol (Ins) phosphate pathway are shown. Mips1S encodes for a Myo-Ins(3)P1 synthase, which converts glucose-6-P to Ins(3)P1. Ins(3)P1 can be produced also by the action of a myo-Ins kinase encoded by Lpa3 gene. Lpa2 encodes an Ins phosphate kinase, which along with other kinases leads to phytic acid, Ins $(1,2,3,4,5,6) \mathrm{P} 6$ synthesis. Lpa1 gene ( $\mathrm{ZmMRP4}$ ) is a transmembrane transporter hypothesised to load phytate into protein storage vacuoles.

mutation, and whose extent appears linked to the phenotype expression. In particular, it has been shown that negative pleiotropic effects lead to lethality in those individuals showing less than $20 \%$ of the wild-type phytate amount (Pilu et al., 2005). Genetic data concerning the heredity of the lpa1-241 trait suggest that an epigenetic phenomenon called paramutation might be involved in this trait (Pilu et al., 2005).

Epigenetic regulation is associated with mitotically and/or meiotically heritable changes in gene expression occurring without changes in DNA sequence. These phenomena are common in eukaryotes and control a number of processes, such as, for example, development, imprinting, transposons and viral sequences silencing, as well as transgene silencing (Martienssen, 1996; Wolffe and Matzke, 1999).

Paramutation is a particular epigenetic phenomenon in which an allele (named paramutagenic) is capable of heritably silencing another allele (paramutable) in trans. Alleles not participating in paramutation are called neutral. So far, paramutation in maize has been studied at four loci: $r 1, b 1, p 1$ and $p l 1$, all involved in the regulation of anthocyanin and flavonoid biosynthesis (reviewed in Chandler et al., 2000). For these genes, reduced pigmentation linked to the paramutated allele correlates with reduced mRNA level and in the case of $b 1$ locus is associated with RNAi phenomena (Das and Messing, 1994; Lund et al., 1995; Chandler et al., 2000; Sidorenko and Peterson, 2001; Della Vedova and Cone, 2004; Chandler and Stam, 2004; Stam and Scheid, 2005; Alleman et al., 2006; Chandler, 2007).

In 1956, Alexander Brink first described paramutation in maize occurring at colored1 $(r 1)$, a complex locus encoding $m y c$-homologous transcription factors that regulate genes involved in the anthocyanin biosynthetic pathway. $R$-r:standard (R-r:std) is a paramutable allele, which produces dark pigmentation of the aleurone. When crossed with paramutagenic allele $R$-stippled $(R-s t)$, in the following generation $R$-r:std shows a variably reduced pigmentation (Brink, 1956). The silenced allele (designated $R-r^{\prime}$ ) is heritable and, like $R$-st, is capable of weak paramutagenic activity for some generations (Brown and Brink, 1960). However, $R-r^{\prime}$ reverts to normal expression $(R-r)$ over subsequent generations, if it is no more exposed to paramutagenic allele $R$-st. In general paramutable alleles of $b 1$ and pl1 loci are unstable, spontaneously changing to the silenced state with high frequency, whereas $p 1$ and $r 1$ epigenetic states are very stable (Chandler et al., 2000; Sidorenko and Peterson, 2001).

Although molecular mechanisms underlying these paramutation phenomena are not fully explained, in some cases, specific DNA sequences such as repeated sequences and/or gene structures are known to correlate with the paramutagenic behaviour of alleles. Paramutation, as well as other gene silencing phenomena, has been found to correlate with changes in DNA methylation and in some cases in chromatin structure.

In this study, we present genetic and molecular analysis indicating that an epigenetic event, like paramutation, may occur at the lpa1 locus; in fact lpa1-241 allele exhibit paramutagenic activity $v$ s the paramutable B73 Lpa1 allele. To our knowledge, this is the first report of a gene silencing phenomenon causing lethality in maize.

\section{Materials and methods}

\section{Nomenclature}

In classical paramutation, silenced (paramutated) alleles are designated with an apostrophe (for example, $\mathrm{Pl}^{\prime}, B^{\prime}$ and so on). In this study, we use apostrophes to distinguish between normally expressing alleles and partially silenced Lpa1 alleles. Thus, Lpa1 alleles have an apostrophe when exposed in trans to lpa1-241 allele for one generation. After two generations of exposure to paramutagenic lpa1-241, the allele has two apostrophes (Lpa1") and so on. When allele genotype can be determined using a ZmMRP4 allele-specific PCR-based molecular marker, a suitable subscript indicating the inbred line is added. Alleles from ACR inbred plants (inbred line homozygous dominant for the colour factors $A 1, C 1$ and $R 1$ ) are named $L p a 1_{A C R}$, whereas alleles from $\mathrm{B} 73$ are $L p a 1_{B 73}$.

\section{Genetic stocks}

The lpa1-241 mutant was originally isolated from the $\mathrm{M}_{2}$ progeny of chemically (ethyl methane sulphonate) mutagenized populations (Pilu et al., 2003). Plants heterozygous for lpa1-241 in the ACR inbred line were used as donors in crosses with the B73 inbred line for five generations and selfed. $\mathrm{BC}_{5} \mathrm{~F}_{2}$ seeds from both pedigrees were used for quantitative analysis of free phosphate, 5-Azacytidine treatment, mips1S gene expression, ZmMRP4 gene expression and methylation analysis. Each selfed generation was also crossed with the homozygous lpa1-1 mutant in B73 background, and seeds were tested for free phosphate content and phenotype segregation; mop 1-1 stock ( $B^{\prime}$ mop1-1/mop11) was provided by the Maize Genetics Cooperation Stock Center (http://maizecoop.cropsci.uiuc.edu/). The lpa1-1 mutant stock was kindly provided by Dr Victor Raboy, USDA ARS, Aberdeen, ID, USA. 
Quantitative free phosphate assay (detection of HIP phenotype)

Individual or pooled seeds were ground in a mortar with a steel pestle and $100 \mathrm{mg}$ of the resulting flour was extracted with $1 \mathrm{ml} 0.4 \mathrm{M} \mathrm{HCl}$ for $1 \mathrm{~h}$ at room temperature. Samples were mixed briefly and $100 \mu$ l were removed and supplemented with $900 \mu \mathrm{l}$ Chen's reagent (6 $\mathrm{N} \mathrm{H}_{2} \mathrm{SO}_{4}: 2.5 \%$ ammonium molybdate: $10 \%$ ascorbic acid: $\mathrm{H}_{2} \mathrm{O}[1: 1: 1: 2, \mathrm{v} / \mathrm{v} / \mathrm{v} / \mathrm{v}]$ ) in microtiter plates (Chen et al., 1956). In these conditions, if phosphate is present, a blue coloured phosphomolybdate complex forms in 1-2 $\mathrm{h}$. After $1 \mathrm{~h}$ at room temperature, the free phosphate content was quantified through the use of a spectrophotometer $(\lambda=650 \mathrm{~nm})$ or evaluated by visual inspection. A $\mathrm{KH}_{2} \mathrm{PO}_{4}$ solution was used as phosphate standard. To test the seeds for HIP phenotype without interfering with their capacity of growth, a small amount of scutellum flour was carefully obtained from a single incision by a hand drill. The flour was extracted in microtiter with $200 \mu \mathrm{l} 0.4 \mathrm{M} \mathrm{HCl}$ for $1 \mathrm{~h}$ at room temperature, and then $800 \mu \mathrm{l}$ of Chen's reagent was added. After $1 \mathrm{~h}$ a Strong HIP phenotype could be detected by visual inspection.

\section{Ipa1 allele molecular genotyping}

Allele genotyping was performed by PCR amplification of a ZmMRP4 sequenced gene portion. Allele-specific primers were designed on a two nucleotides insertion polymorphism found in the ACR ZmMRP4 10th intron. ACR specific primer was ZmMRP4 + 6092Ra (5'-AATC AAGACGATGAGAAAAGTTAT-3'), whereas B73 specific primer was ZmMRP4 + 6092Rb (5'-AATCAAGACG ATGAGAAAAGTTC-3'). ACR allele-specific amplifications were performed in a reaction mix containing an aliquot of genomic DNA, 1X Green Go Taq buffer, $2.5 \mu \mathrm{M}$ $\mathrm{MgCl}_{2}, 0.2 \mu \mathrm{M}$ each of dATP, dCTP, dGTP and dTTP, $0.3 \mu \mathrm{M}$ of reverse $\mathrm{ZmMRP} 4+6092 \mathrm{Ra}$ ACR-specific primer, $0.3 \mu \mathrm{M}$ of forward ZmMRP4 $+5590 \mathrm{~F}$ primer (5'-TGGGAATGTGGTTTCTTAATGC-3') and 1.25 unit of Go Taq Flexy DNA polymerase (Promega, Madison, WI, USA), in a final volume of $25 \mu \mathrm{l}$. The reaction mix underwent an initial denaturation step at $94^{\circ} \mathrm{C}$ for $2.5 \mathrm{~min}, 37$ cycles of denaturation at $94^{\circ} \mathrm{C}$ for $45 \mathrm{~s}$, annealing at $63^{\circ} \mathrm{C}$ for $1 \mathrm{~min}$, extension at $72^{\circ} \mathrm{C}$ for $1.5 \mathrm{~min}$. Extension at $72{ }^{\circ} \mathrm{C}$ for $5 \mathrm{~min}$ was performed to complete the reaction. The ACR allele-specific amplification product is $498 \mathrm{bp}$ long. B73 allele-specific amplifications were performed in a reaction mix identical to that of ACR allele-specific amplifications, except that $0.3 \mu \mathrm{M}$ of $\mathrm{ZmMRP} 4+6092 \mathrm{Rb}$ B73-specific primers were used instead of ZmMRP4 + 6092Ra. The reaction mix underwent an initial denaturation step at $94{ }^{\circ} \mathrm{C}$ for $2.5 \mathrm{~min}$, 36 cycles of denaturation at $94^{\circ} \mathrm{C}$ for $45 \mathrm{~s}$, annealing at $65^{\circ} \mathrm{C}$ for $1 \mathrm{~min}$, extension at $72^{\circ} \mathrm{C}$ for $1.5 \mathrm{~min}$. Extension at $72{ }^{\circ} \mathrm{C}$ for $5 \mathrm{~min}$ was performed to complete the reaction. The B73 specific amplificate is $503 \mathrm{bp}$ long. Amplification products were visualized on $1.5 \%(\mathrm{w} / \mathrm{v})$ agarose gels with ethidium bromide staining.

\section{5-aza-2'-deoxycytidine (azacytidine) treatment}

Mature dry seeds were sterilized with 5\% sodium hypochlorite for $15 \mathrm{~min}$ and then incubated with $20 \mathrm{ml}$ of $30 \mu \mathrm{M}$ 5-aza-2'-deoxycytidine (Sigma, product No. A3656, St Louis, Mo, USA) solution in rotating flasks at $28^{\circ} \mathrm{C}$ for $15 \mathrm{~h}$ (in order to obtain mature plants), or at $30^{\circ} \mathrm{C}$ for $18 \mathrm{~h}$ (for embryo rescue and seedlings analysis). Control seeds were incubated with $20 \mathrm{ml}$ deionised water under the same conditions.

\section{Embryo rescue}

5-Azacytidine-treated embryos were removed aseptically and transferred to Murashige and Skoog salt mixture (pH 5.6; Sigma, St Louis, MO, USA, product no. M5519) containing $2 \%$ sucrose, solidified with $0.8 \%$ agar (Plant agar, Duchefa, Haarlem, The Netherlands). Cultures were incubated in a growth chamber at $25^{\circ} \mathrm{C}$ with a $18 / 6$ light/dark photoperiod. Seedling elongation was measured after 6-14 days and shoot tissue was sampled and stored at $-80^{\circ} \mathrm{C}$ for subsequent DNA and RNA extraction. The light source consisted of four cool white (F36T12/CW/HO) fluorescent lamps from GTE SYLVANIA (Lighting Products Group, Danvers, MA, USA). This experiment was conducted on a total of 20 treated lpa1-241/lpa1-241, 23 untreated lpa1-241/lpa1-241, 33 treated wild type, 38 untreated wild type, 26 treated lpa1-1/lpa1-1 and 26 untreated lpa1-1/lpa1-1 individuals.

\section{Reverse transcriptase-PCR expression analysis}

Total RNA was extracted from frozen shoots of 6-7 days old wild type, or 14-15 days old selected strong HIP phenotype seeds using the method described by van Tunen et al. (1988). RT-PCR was used to detect mips1S and ZmMRP4 gene transcripts. First strand cDNA was synthesized with an oligo (dT) primer from total RNA extracted from shoots. All RNA samples were treated with DNase $\left(1{\left.\mathrm{U} \mu \mathrm{g}^{-1}\right)}^{-1}\right.$ before cDNA synthesis. Firststrand cDNA was used as the template for PCR amplification. Amplification reactions containing an aliquot of cDNA synthesized from $5 \mu \mathrm{g}$ of total RNA, 1X Green Go Taq buffer, $2.5 \mu \mathrm{M} \mathrm{MgCl} 2,0.2 \mu \mathrm{M}$ each of dATP, dCTP, dGTP and dTTP, $0.3 \mu \mathrm{M}$ of each primer and 1.25 U of Go Taq Flexy DNA polymerase (Promega) were performed in a final volume of $25 \mu \mathrm{l}$. The reaction mix underwent 34 cycles of denaturation at $94{ }^{\circ} \mathrm{C}$ for $45 \mathrm{~s}$, annealing at $62^{\circ} \mathrm{C}$ for $1 \mathrm{~min}$, extension at $72^{\circ}$ for $1.5 \mathrm{~min}$. Extension at $72{ }^{\circ} \mathrm{C}$ for $5 \mathrm{~min}$ was performed to complete the reaction. A set of primers specific for the orange pericarp 1 (orp1) gene, which encodes the $\beta$-subunit of tryptophan synthase (Wright et al., 1992), was used to standardize the concentration of the different samples. orp1-specific sequences were amplified using the following primers: the upstream primer, $5^{\prime}$-AAGGACGTGCA CACCGC-3' and downstream primer, 5'-CAGATACAGA ACAACAACTC- $3^{\prime}$. The length of the amplified product was $207 \mathrm{bp}$. Several cycles of successive cDNA dilutions and orp 1 amplification were carried out to obtain similar amplification signals in the different samples. For mRNA detection of the mips1S gene under analysis, the following specific primer sets were used: Zm1302 (upstream primer $5^{\prime}$-GCTCTTGGCTGAGCTCAGCA-3') and Zm1580 (downstream primer 5'-GTTCCCTTCCAGCAG CTAAC-3'). The amplified product was $279 \mathrm{bp}$. ZmMRP4 mRNA detection was conducted with specific primers designed on ZmMRP4 genomic sequence (Shi et al., 2007): ZmMRP4 + 5135F (upstream primer 5'-TCATGG TGTAAGTTGTATGTTTC-3') and ZmMRP4+6206R (downstream primer $5^{\prime}$-CTTCTCTATATACAGCTCGAC-3'). A $677 \mathrm{bp}$ amplificate is obtained after 33 cycles of 
denaturation at $94{ }^{\circ} \mathrm{C}$ for $45 \mathrm{~s}$, annealing at $60{ }^{\circ} \mathrm{C}$ for $1 \mathrm{~min}$, extension at $72{ }^{\circ} \mathrm{C}$ for $1.5 \mathrm{~min}$. Final extension at $72{ }^{\circ} \mathrm{C}$ for $5 \mathrm{~min}$ was performed. Each expression analysis was conducted on RNA extracted from five individuals, in three replicates at least.

PCR products were loaded on $2 \%(\mathrm{w} / \mathrm{v})$ agarose gels and visualized by ethidium bromide staining under ultraviolet light.

\section{Results}

Inheritance of Ipa1-241 trait: Ipa1-241 allele may cause partial Lpa1 allele silencing

To quickly follow the lpa1 trait segregation, we scored for the seed free inorganic phosphate content using Chen's assay performed in microtitre plates (Chen et al., 1956; Raboy et al. 2000; Pilu et al. 2003). We defined four phenotypic classes corresponding to the level of seed free inorganic phosphate expressed as milligram of atomic $\mathrm{P}$ per gram of flour. These classes, wild type $(0-0.3)$, weak (0.3-0.5), intermediate (0.5-1.4), strong ( $>1.4)$, are easily scored by visual inspection if the assays are performed in microtiter plates (Figure 2). Furthermore, we used the ZmMRP4 gene sequence data to produce an allelespecific PCR-based molecular marker to discriminate the lpa1-241 ACR allele from Lpa1 B73 allele and follow the allele segregations showed in Figure 3.

As shown in Figure 3 where we report a schematic pedigree of the lpa1-241 trait, the original lpa1-241 mutation event occurred in an ACR inbred line (Figure 3, arrow 1). The mutant phenotype was observed in $F_{2}$, where the strong HIP class segregated 1:3 as expected for a recessive mutation (Figure 3 , cross 2 ). In this cross, heterozygotes showed a weak phenotype, although, as shown in our previous work, crosses to wild-type ACR plants resulted in 100\% wild-type progeny (Figure 3, cross 3 ). Thus, we originally speculated that the mutation was not completely recessive (Pilu et al. 2003) and later on conjectured (Pilu et al., 2005) there might be some kind of allelic interaction such as paramutation causing a silencing of the wild-type Lpa1 allele. With the aim of better understanding of this behaviour, we crossed heterozygous ACR Lpa1/lpa1-241 families with wildtype plants from the B73 inbred line and observed that

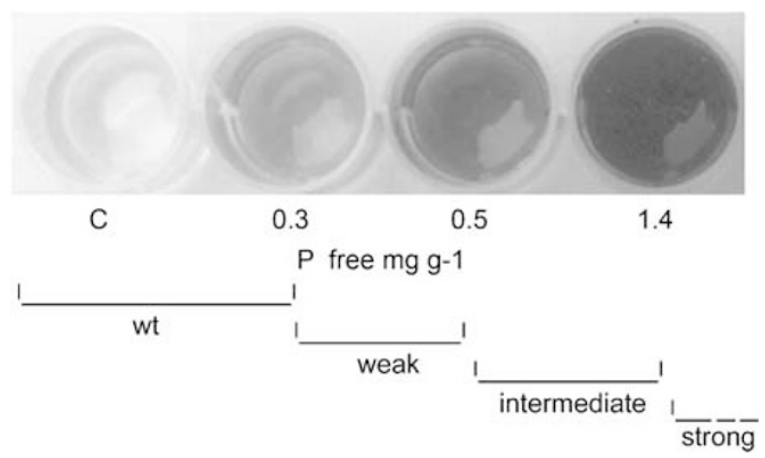

Figure 2 Assay to detect lpa1-241 phenotypic classes. Single seeds were crushed, extracted and assayed for free P using a microtitre plate-based colorimetric assay (Chen et al., 1956). Classes detectable by visual inspection were: wild type (wt) $0-0.3$; weak (W) $0.3-0.5$; intermediate (I) $0.5-1.4$; strong (S) $>1.4$, expressed as milligram of atomic P per gram of flour. only the wild-type phenotype was detected in $F_{1}$ (Figure 3, cross 4). Selfing heterozygous Lpa1 $_{\mathrm{B} 73} /$ lpa1$241_{\mathrm{ACR}}$ plants, we obtained a segregating $\mathrm{F}_{2}$ generation (Figure 3, cross 5) in which the phenotype classes are similar to those obtained in segregant $\mathrm{F}_{2}$ ACR seeds.

Furthermore, in $F_{3}$ seeds (Figure 3, cross 6), we observed a general decrease in the size of wild-type and weak classes, associated to a general increase of intermediate and strong classes. The segregation data regarding $\mathrm{F}_{2}, \mathrm{~F}_{3}$ and $\mathrm{F}_{4}$ families showed a consistent increase of strong/intermediate HIP phenotype, which was correlated to the number of selfings (Figure 4a and Table 1). Furthermore, no progeny was obtained from lpa1-241 $\mathrm{ACR}$ homozygotes because of the negative pleiotropic effects associated to the strong lpa phenotype.

This non-mendelian segregation could be explained with a progressive Lpa1 allele silencing occurring in lpa1241 families over subsequent generations.

To verify these data, we crossed several heterozygous plants of subsequent generation Lpa1 $_{\mathrm{B} 73} /$ lpa1-241 $1_{\mathrm{ACR}}$ Lpa1' ${ }_{\mathrm{B} 73} /$ lpa1-241 ${ }_{\mathrm{ACR}}$ and Lpa1" ${ }_{\mathrm{B} 73} /$ lpa1-241 $_{\mathrm{ACR}}$ to homozygous recessive stable lpa1-1 line. In these crosses, we expected a segregation ratio of 1:1 for lpa1 phenotype as expected for a backcross of monogenic recessive mutation. However, even in this case, phenotype segregation of the progeny showed a decrease in the size of wild-type and weak classes and a correlated increase of intermediate and strong classes associated to progressive exposure of the Lpa1 allele to the paramutagenic lpa1-241 (Table 2 and Figure 4b).

\section{Paramutated Lpa1 allele reverts to wild type}

Segregating families carrying a partially silenced Lpa1' allele showed a weak phenotype (Figure 3, cross 7), but in subsequent generations of selfing, we observed a reversion to wild type in the absence of the lpa1-241 allele.

In line with this, measuring the seed free phosphate amount in partially silenced Lpa1'/Lpa1' progeny (Figure 3, cross 8), we detected a progressive reversion to wild-type phenotype: $L p a 1^{\prime} / L p a 1^{\prime}$ seed phenotype from a $\mathrm{F}_{2}$ segregant family resulted in $0.50 \mathrm{mg}$ of atomic $\mathrm{P}$ per gram of flour in average, whereas subsequent progeny from these homozygous Lpa1' plants produced seeds with lower free phosphate levels: $0.20 \mathrm{mg} \mathrm{g}^{-1}$ for $\mathrm{F}_{3}$ and $0.14 \mathrm{mg} \mathrm{g}^{-1}$ for $\mathrm{F}_{4}$ seeds.

The same behaviour was observed in homozygous

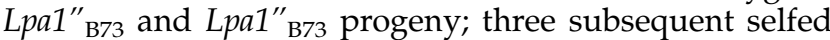
generations were produced and when the average seed free phosphate content was measured in each generation, a steady decrease was observed (data not shown).

\section{Ipa1-241 exhibits silencing activity over different alleles} To directly detect $L p a 1_{\mathrm{B} 73}$ allele silencing, heterozygous Lpa1 $_{\mathrm{B} 73} /$ lpa1-241 $\mathrm{F}_{1}$ plants were crossed with homozygous lpa1-1/lpa1-1 plants in B73 background, and the seed free phosphate content of the progeny was assayed (Figure 5a). A 1:1 segregation ratio between strong and weak phenotype, indicating a partial silencing of $L p a 1_{\mathrm{B} 73}$ allele (weak phenotype), was obtained. As a control, the progeny of the cross between B73 wild-type plants and B73 lpa1-1 plants was assayed and only wild-type seeds were obtained (Figure 5b). 


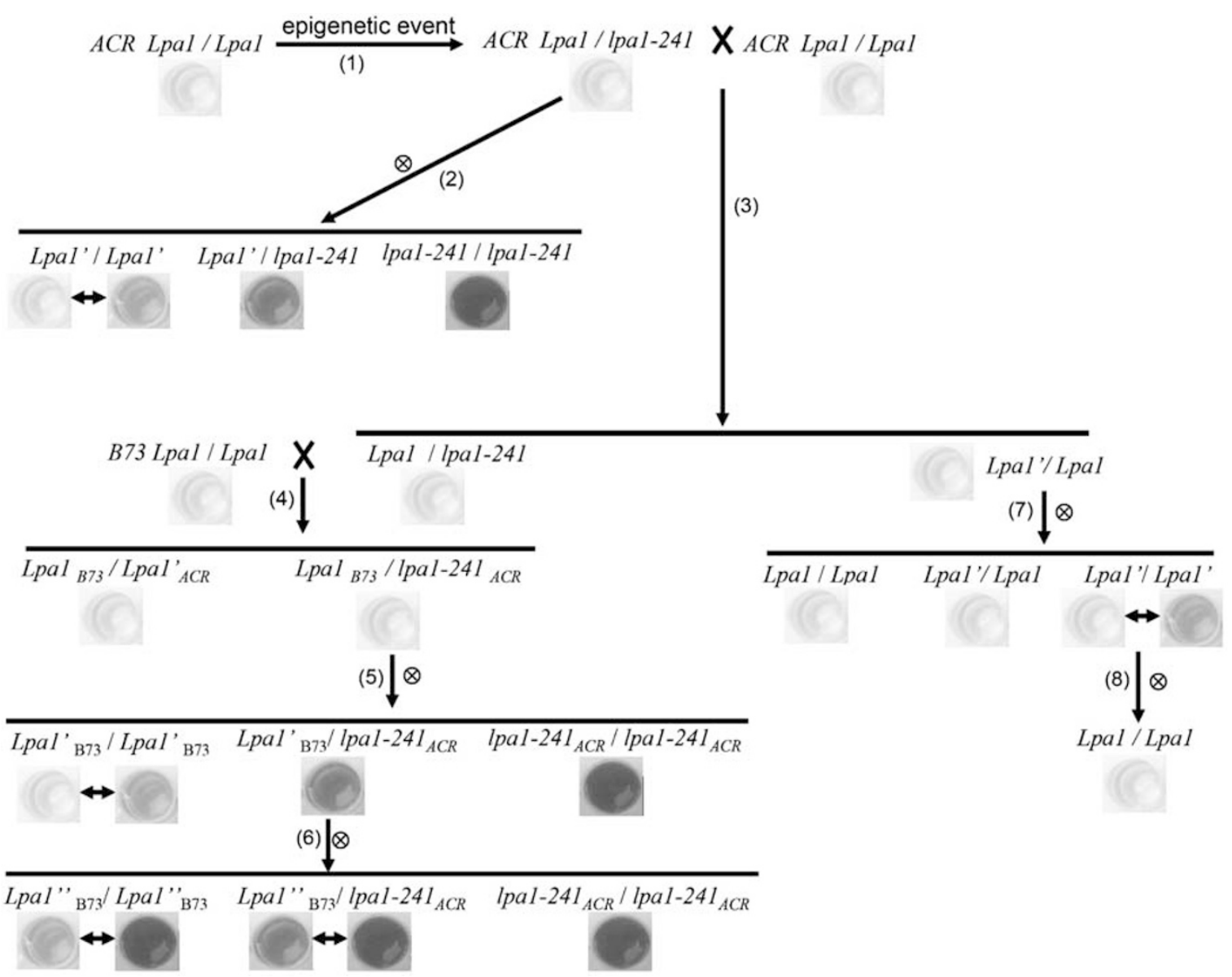

Figure 3 Diagram of lpa1-241 mutation pedigree. A Lpa1 wild-type allele spontaneously underwent silencing in ACR inbred line carrying the colour factors A1,C1 and R1 (1). The event was named lpa1-241 and its phenotype was detected in the progeny obtained by selfing Lpa1/lpa1241 ACR plant (2). Lpa1/lpa1-241 ACR heterozygous was also crossed with Lpa1/Lpa1 ACR line (3) and the heterozygous Lpa1/lpa1-241 ACR obtained was crossed to a wild-type B73 inbred line (4). Segregating phenotypes obtained in subsequent generations of selfing are shown (5 and 6). Phenotypes and genotypes of the progeny of a weakly silenced allele Lpa1' are also shown (7, 8). A free P microtiter plate-based colorimetric assay (Chen et al., 1956) associated with genotype is also shown. Genotypes have been determined by specific ZmMRP4 allele molecular marker.

The same experiment was conducted with plants from W64A, K6, H99 and W22 inbred lines. An $\mathrm{F}_{1}$ family of each genotype, Lpa1 ${ }_{\mathrm{W} 64 \mathrm{~A}} /$ lpa1-241, Lpa1 $1_{\mathrm{K} 6} /$ lpa1-241, Lpa1 $_{\mathrm{H} 99} /$ lpa1-241 and Lpa1 $1_{\mathrm{W} 22} /$ lpa1-241 $\mathrm{F}_{1}$ was crossed with a B73 lpa1-1/lpa1-1 family. Each cross progeny was analysed by visual inspection and found to segregate 1:1 for strong and weak phenotype, indicating that the expression of every tested allele is reduced after one generation in trans with the lpa1-241 $A C R$ allele. As negative controls, wild-type plants from each inbred line were crossed with a B73 lpa1-1/lpa1-1 family and the progeny assayed, only wild-type seeds were obtained.

\section{Lpa1 gene undergoes spontaneous silencing with high frequency}

To estimate the frequency of spontaneous occurrence of lpa1 mutation, B73 homozygous lpa1-1 mutant lines were crossed with wild-type Lpa1/Lpa1 B73 plants and the progenies were screened for the strong free phosphate phenotype. We used lpa1-1 mutation as a tester to detect the phenotypic expression level of possible silenced Lpa1' epialleles as well as to evaluate the spontaneous frequency of occurrence of lpa1 mutation because it has a stable, strong HIP phenotype and is viable in homozygosity (Raboy et al., 2000).

About $2500 \quad F_{1}$ seeds were assayed and 13 seeds displaying strong silencing were detected, indicating novel events of Lpa1 gene silencing. Thus, the frequency of spontaneous silencing of Lpa1 allele resulted $5.2 \times 10^{-3}$.

\section{Lpa1 gene silencing is not affected by mop 1-1}

The wild-type gene mediator of paramutation1-1 (Mop1-1) is required for establishment and maintenance of paramutation at several maize loci and the mop 1-1 recessive mutation affects paramutation (Dorweiler et al., 2000), so we performed a test to assay the behaviour of our lpa1 mutation. We used a genotype carrying $B^{\prime}$ allele so as to be able to follow in the offspring, the purple plants homozygous for mop1-1/mop-1.

Heterozygous Lpa1/lpa1-241 families were crossed with mop1-1 $B^{\prime}$ plants and the progenies were selfed. $B^{\prime} /$ - coloured plants present in the progeny were homozygous mop1-1/mop1-1. 27 coloured (mop1-1/mop1-1 $\left.B^{\prime} /-\right)$ and 18 colourless/light (Mop1-1/- $\left.B^{\prime} /-\right)$ plants used as control were self-fertilized and their progenies assayed for HIP phenotype (Figure 6). HIP phenotype 

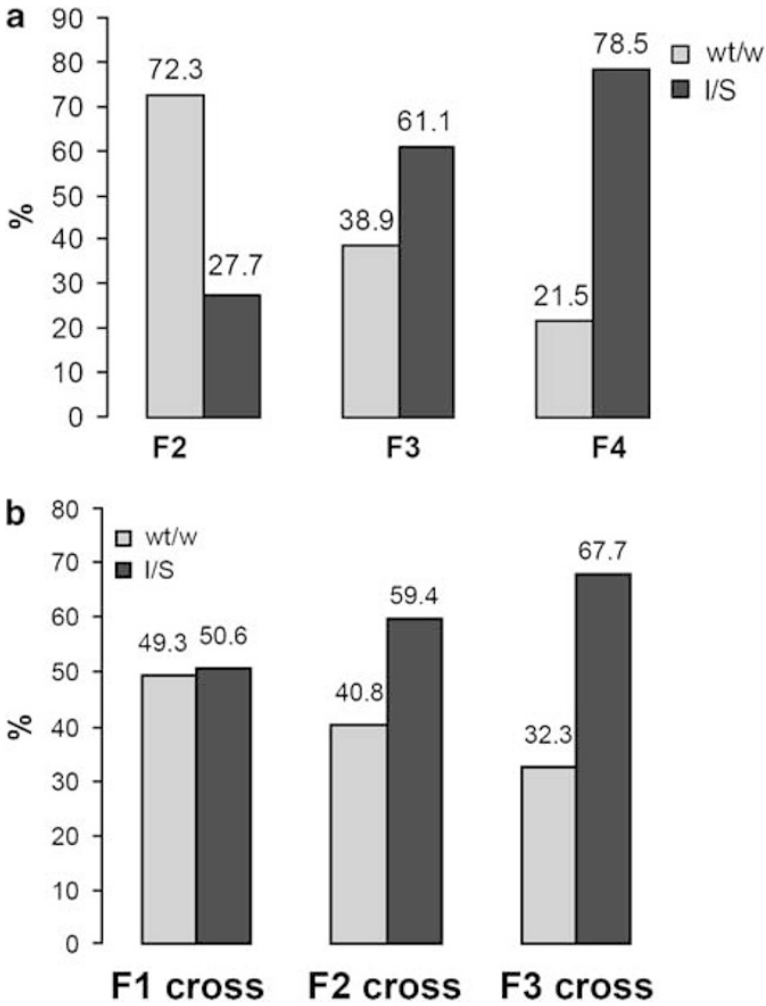

Figure 4 Changes in phenotypic class segregation ratios over generations in the presence of lpa1-241 allele. (a) Seed free phosphate phenotypic classes are presented in histogram as a percentage of occurrence in selfed heterozygous progenies, where $\mathrm{F}_{2}$ refers to Lpa1/lpa1-241 selfed progeny, $\mathrm{F}_{3}$ refers to Lpa1'/lpa1-241 selfed progeny and $\mathrm{F}_{4}$ refers to Lpa1"/lpa1-241 selfed progeny. (b) Histogram representation of phenotypic classes segregation ratios in the progenies of the same families crossed with a homozygous lpa1-1 recessive mutant: $F_{1}$ cross refers to Lpa1/lpa1-241 × lpa1-1/ lpa1-1 progeny, $F_{2}$ cross refers to Lpa1'/lpa1-241 $\times$ lpa1-1/lpa1-1 progeny, $\mathrm{F}_{3}$ cross refers to $L p a 1^{\prime \prime} /$ lpa1-241 × lpa1-1/lpa1-1 progeny. The sum of wild-type and weak $(\mathrm{wt} / \mathrm{w})$ phenotype occurrence (grey bars) vs intermediate plus strong (I/S) phenotype occurrence (black bars) are shown. Percentage values are shown at the top of each bar. was found in both coloured and colourless plants, indicating that the mop1-1 mutation is unable to modify the lpa1-241 phenotype.

\section{Seed treatment with 5-Azacytidine partially reverts HIP phenotype and reduces pleiotropic effects in Ipa1-241 mutant seedling}

Two different experiments with the demethylating agent 5-Azacytidine were conducted on lpa1-241 mutants, the first experiment concerning the study of the effect of 5-Azacytidine seed treatment on the HIP offspring phenotype, and the second one regarding the effect of this treatment on the pleiotropic effects on the seedling caused by lpa1-241 mutation.

A significant reduction of the phenotype strength was found in lpa1-241/lpa1-241 individuals obtained from self-pollinated plants derived from 5-aza-2'-deoxycytidine-treated seeds compared with untreated controls (Figure 7). By contrast, neither seeds produced by treated wild-type B73 seeds nor homozygous lpa1-1 seeds (data not shown) revealed significant differences in respect to untreated controls (Figure 7).

Seeds expressing the strong HIP phenotype are unable to germinate, but slow growing seedlings can be obtained if embryos are rescued in vitro on Murashige and Skoog (MS) medium. These seeds treated with 5-Azacytidine always showed a remarkable increase in growth (of about $50 \%$ ) compared with untreated controls of the same phenotypic class (Figure 8a). No significant difference was detected between treated and untreated Lpa1/Lpa1 B73 (Figure 8b) or lpa1-1 homozygote seedlings (not shown in figure).

The demethylating agent 5-Azacytidine partially restores ZmMRP4 (Lpa1 gene) as well as mips1s gene expression After treatment of seeds with 5-Azacytidine, mips1s and ZmMRP4 gene expression levels were analysed in seedling tissues by RT-PCR.

Table 1 Effects of generation on heritability of high inorganic phosphate phenotype (HIP) phenotype in lpa1-241 lines

\begin{tabular}{|c|c|c|c|c|c|}
\hline \multirow[t]{2}{*}{ Cross } & \multirow[t]{2}{*}{ Generation } & \multicolumn{4}{|c|}{ Phenotypic classification } \\
\hline & & $W t$ & $W$ & $I$ & $S$ \\
\hline$L p a 1_{B 73} / l p a 1-241$ selfed & $\mathrm{F}_{2}$ & $45(31.9 \%)$ & $57(40.4 \%)$ & $8(5.7 \%)$ & $31(22 \%)$ \\
\hline 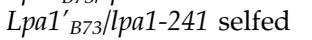 & $\mathrm{F}_{3}$ & $39(24.1 \%)$ & $24(14.8 \%)$ & 39 (24.1) & $60(37 \%)$ \\
\hline Lpa1" $_{B 73} / l p a 1-241$ selfed & $\mathrm{F}_{4}$ & $2(1.9 \%)$ & $21(19.6 \%)$ & $65(60.7 \%)$ & $19(17.8 \%)$ \\
\hline
\end{tabular}

The seeds obtained were assayed for free P using a microtiter plate-based colorimetric assay (Chen et al., 1956) and visually classified as wild type (wt), weak (W), intermediate (I) and strong (S).

Table 2 Effects of generation on heritability of high inorganic phosphate phenotype (HIP) phenotype in lpa1-241 lines

\begin{tabular}{|c|c|c|c|c|c|}
\hline \multirow[t]{2}{*}{ Cross } & \multirow[t]{2}{*}{ Generation } & \multicolumn{4}{|c|}{ Phenotypic classification } \\
\hline & & $W t$ & W & $I$ & $S$ \\
\hline $\operatorname{Lpa}_{\text {B7з/lpa1-241 X lpa1-1/lpa1-1 }}$ & $\mathrm{F}_{1}$ & $65(30.5 \%)$ & $40(18.8 \%)$ & $8(3.7 \%)$ & $100(46.9 \%)$ \\
\hline 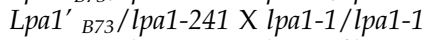 & $\mathrm{F}_{2}$ & $45(24.9 \%)$ & $29(15.9 \%)$ & $20(10.8 \%)$ & $90(48.6 \%)$ \\
\hline${ }_{\text {Lpa1" }}{ }_{\text {B73/lpa1-241 X lpa1-1/lpa1-1 }}$ & $\mathrm{F}_{3}$ & $9(9.4 \%)$ & $22(22.9 \%)$ & $21(21.9 \%)$ & $44(45.8 \%)$ \\
\hline
\end{tabular}

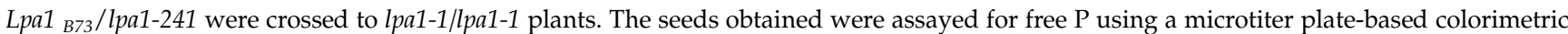
assay (Chen et al., 1956) and visually classified as wild type (Wt), weak (W), intermediate (I) and strong (S). 
As previously reported in our studies on lpa1-241 mutation (Pilu et al., 2003), mips1s expression is reduced in untreated strong HIP phenotype-expressing mutants compared with the untreated wild type (Figure 8c, lane 1 vs lane 3). A detectable increase in mips1s expression was instead found in treated lpa1-241/lpa1-241 strong HIP phenotype mutants compared with untreated mutants of the same phenotypic class (Figure 8c, lane 3 vs lane 4),

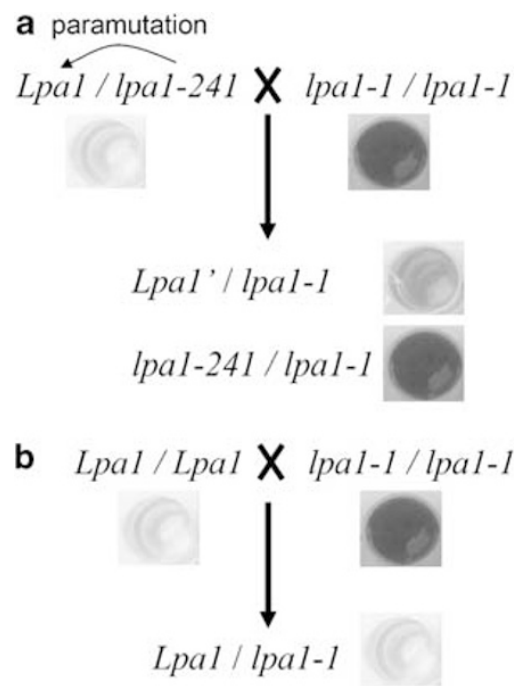

Figure 5 Lpa1 allele silencing test after exposure to lpa1-241 paramutagenic allele. Heterozygous Lpa1/lpa1-241 families have been used in crosses with homozygous lpa1-1 mutant as a test for Lpa1 alleles activity. (a) Phenotypes obtained crossing lpa1-1/lpa1-1 with a Lpa1/lpa1-241 $\mathrm{F}_{1}$ family. Curved arrow indicates the silencing effect of lpa1-241 allele on the Lpa1 allele causing silencing (Lpa1'). (b) Seed free phosphate phenotype of wild-type Lpa1/Lpa1 line crossed with lpa1-1/lpa1-1. whereas no detectable differences were found between treated and untreated wild type (Figure 8c, lane 1 vs lane 2). ZmMRP4 gene expression shows a similar pattern to that of mips1s. Untreated mutants show a lower expression compared with untreated wild type (Figure 8c, lane 1 vs lane 3), and, again, treated mutants expression level increases after 5-Azacytidine treatment (Figure 8c, lane 3 vs lane 4$)$.

Reverse transcriptase-PCR analysis performed on lpa1-1 homozygote seedlings carried out in the same experimental conditions showed no significant variation in the expression of both mips1s and ZmMRP4 genes (Figure 8d).

\section{Discussion}

The lpa1-241 recessive mutation was isolated in an ethyl methane sulphonate mutagenesis ACR inbred line showing a strong HIP phenotype in the seed. A first evidence of non-Mendelian inheritance of lpa1 trait came from the appearance of unexpected free phosphate phenotypes in $\mathrm{Lpa1}_{A C R} /$ lpa1-241 $1_{A C R}$. When heterozygous families were selfed, we observed an overall increase of the mutant phenotype ratio due to the appearance of weak and intermediate phenotype, not consistent with a monogenic recessive mutation (Figure 3 , cross 2 ). This phenomenon can be explained with a partial Lpa1 allele silencing caused by trans interaction with the paramutagenic lpa1-241 allele.

To prove this hypothesis, we used an allele-specific PCR-based molecular marker to discriminate the lpa1-241 ACR allele from Lpa1 B73 allele and follow the alleles segregations. More accurate evidences were obtained by selfing lpa1-241/Lpa1 ${ }_{B 73}$ heterozygotes (Figure 3, crosses 5 and 6).
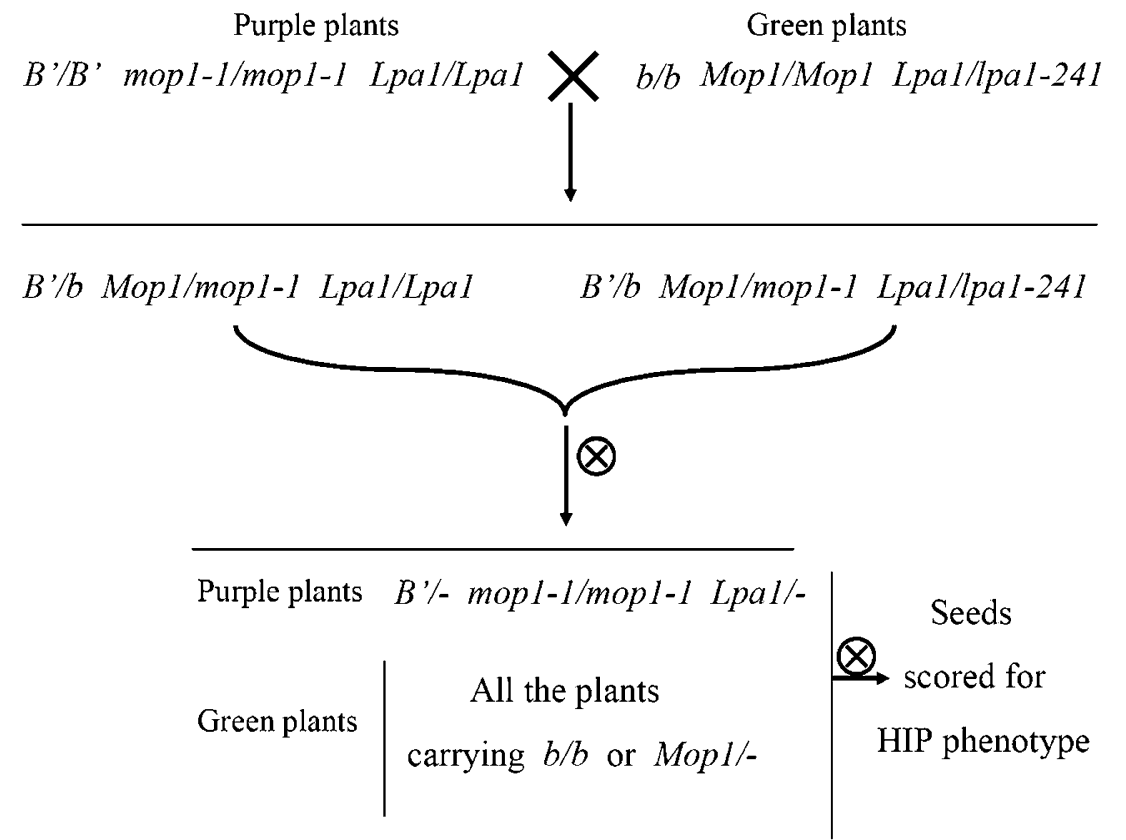

Figure 6 Diagram showing the crosses between mop1-1 and lpa1-241 mutants and the genotypes tested. Homozygous mop1-1 purple plants ( $B^{\prime} / B^{\prime}$ mop1-1/mop1-1 Lpa1/Lpa1) were crossed with heterozygous lpa1-241 green plants (b/b Mop1/Mop1 Lpa1/lpa1-241), the progeny was selfed and seeds obtained were planted. Purple plants $\left(B^{\prime} /-\right.$ mop1-1/mop1-1 Lpa1/-) were then selfed and the seeds obtained scored for inorganic phosphate content. Green plants were used as control. 
As observed in ACR families, in $F_{2}, F_{3}$ and $F_{4}$ generations where lpa1-241 allele was present, seed free phosphate levels increased, suggesting a reduction in the activity of the $L p a 1_{B 73}$ allele (Table 1 and Figure $4 a$ ). After some generations, selfed families reached a level of phenotype expression whose pleiotropic effects seriously impair fitness. Thus, no further progeny can be obtained

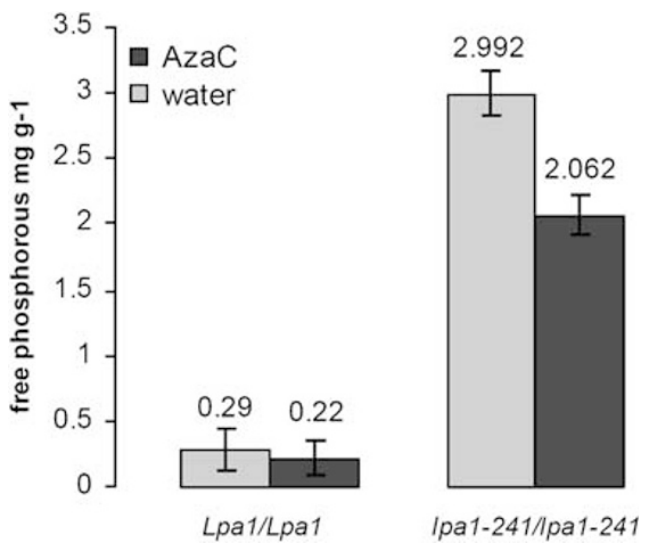

Figure 7 Seed free phosphorus content of 5-Azacytidine-treated lpa1-241 seeds progeny. Average seed free phosphorus content (milligram P per gram of flour) of Lpa1/Lpa1 B73 and homozygous lpa1-241/lpa1-241 individuals from lpa1-241 segregating families. Black bars refer to 5-Azacytidine-treated seeds; grey bars refer to untreated controls. Confidence intervals at $95 \%$ are shown. from such plants. In addition, the progressive Lpa1 silencing (Lpa1' Lpa1", and so on) could be also detected by crossing plants from each generation with homozygous lpa1-1: where a 1:1 segregation ratio is expected, we scored mutant seeds in more than $50 \%$ of the cases (Table 2 and Figure $4 \mathrm{~b}$ ).

A common aspect in gene silencing phenomena such as some cases of classical paramutation is that spontaneous silencing can occur with high frequency. We found that silenced lpa1 alleles showing the strong HIP phenotype can spontaneously occur in B73 line with a frequency of more than $10^{-3}$.

Collected genetic and phenotypic data regarding the hereditability of lpa1-241 locus are compatible with a gene silencing phenomenon such as paramutation. So far, paramutation in maize has been studied at four loci $r 1, b 1, p l 1$ and $p 1$, all involved in flavonoids and anthocyanins biosynthesis (reviewed in Chandler et al., 2000).

The booster1 (b1) locus contains a single coding region for a transcription factor that regulates synthesis of anthocyanin pigments in many epidermal tissues. The active, paramutable B-I (Booster-Intense) allele spontaneously becomes partially silent $\left(B^{\prime}\right)$ with high frequency. Crossing an active $B-I$ with a $B^{\prime}$ causes the downregulation of $B-I$, which also acquires paramutagenic activity (Coe, 1959, 1966).

In 1995, paramutation was discovered at purple plant1 (pl1) locus, another anthocyanin pathway regulator. In
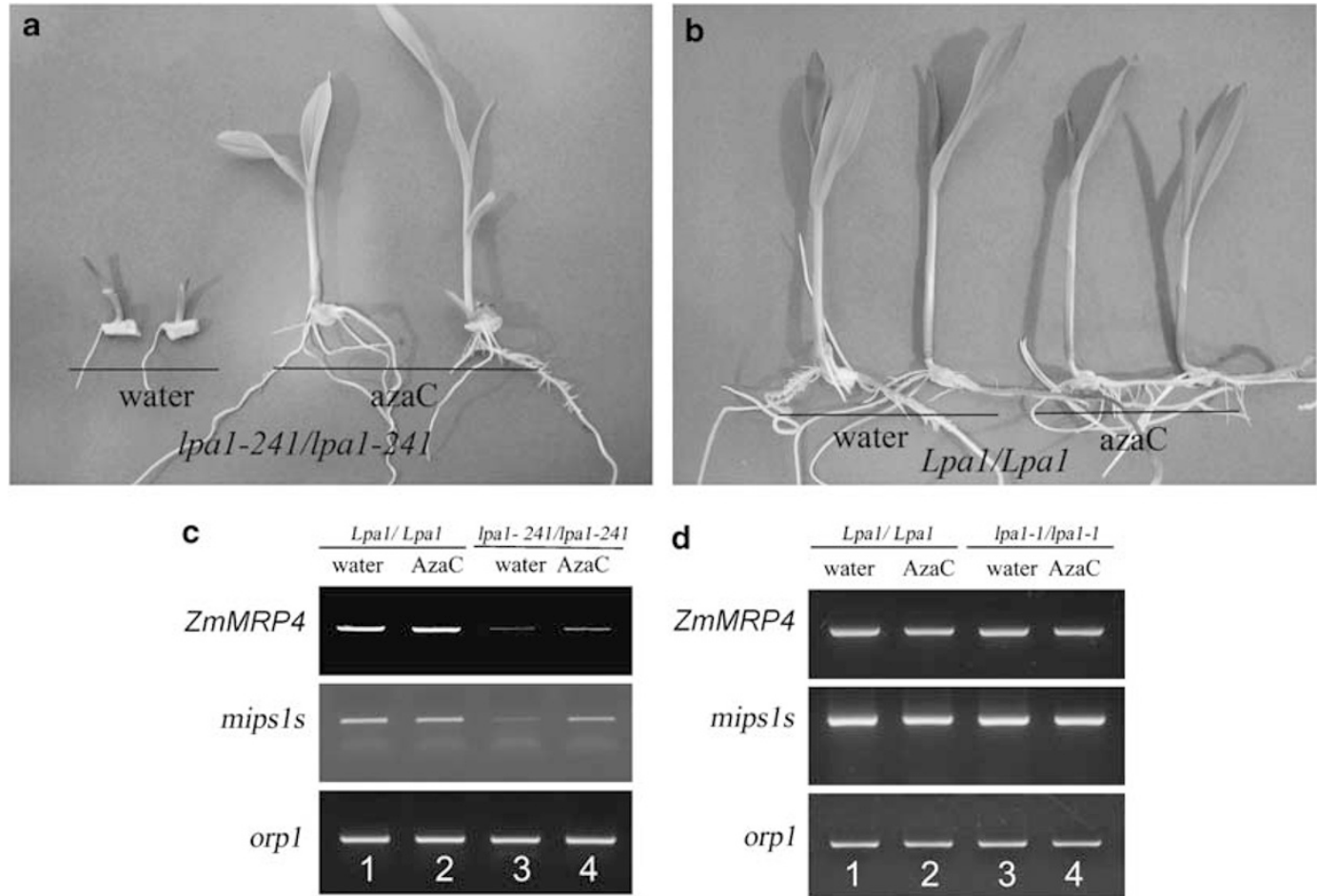

Figure 8 Effects of seeds 5-Azacytidine treatment on lpa1-241 seedlings growth in vitro and on mips1s and ZmMRP4 genes expression. (a) Fourteen days old homozygous lpa1-241 seedlings, untreated (water) and treated (AzaC). (b) Six days old Lpa1/Lpa1 B73 seedlings, untreated (water) and treated (AzaC). Plants were obtained by embryos removed aseptically from the seeds previously treated and transferred to Murashige and Skoog tissue culture medium. (c) Reverse transcriptase-PCR (RT-PCR) analysis showing the expression of ZmMRP4 and mips1s gene in wild-type (untreated, lane 1; treated, lane 2) and homozygous lpa1-241 (untreated, lane 3; treated, lane 4). Orp1 gene amplification is shown as control. (d) RT-PCR analysis of expression of ZmMRP4 and mips1s gene in wild-type (untreated, lane 1; treated, lane 2) compared with homozygous lpa1-1 (untreated, lane 3; treated, lane 4). Orp1 gene amplification is also shown. 
this case, the paramutable allele Pl-Rhoades $(P l-R h)$ is silenced when exposed in trans to its spontaneously derived, silenced paramutagenic $P l^{\prime}$ allele (Hollick et al. 1995).

At $p 1$ (pericarp color1) locus, two spontaneous epialleles (P-pr-1 and $P-p r-2)$ were isolated. They showed moderate stability and weak paramutagenic effect on $P-r r$ allele (Das and Messing, 1994). In addition, a heritable, paramutagenic $P-r r^{\prime}$ silenced allele arises by transgeneinduced silencing (Sidorenko and Peterson, 2001). This locus controls the expression of phlobaphene pathway in several tissues, including pericarp and cob.

DNA methylation is known to correlate with epigenetic gene silencing, and epigenetic gene silencing is known to occur by repression of transcription (TGS, transcriptional gene silencing) or by affecting mRNA stability (PTGS, post-transcriptional gene silencing). TGS is associated to chromatin remodelling processes, such as histones modification, substitutions and DNA methylation (Grant-Downton and Dickinson, 2005), whereas PTGS is mediated by various classes of small RNAs (Vaucheret, 2006). Small RNA pathways also act on DNA, mediating chromatin remodelling or even sequence elimination (Vaucheret, 2006). With the aim of understanding whether DNA methylation is involved in our trait, we performed experiments with the demethylating agent 5-aza-2'-deoxycytidine. Interestingly, we found that treated seeds yielded in the next generation homozygous mutant seeds with a significant reduction, compared with control, in free phosphate (Figure 7). No significant variation was found for wild-type B73 used as control or lpa1-1/lpa1-1 seeds (data not shown). Also, selected lpa1-241/lpa1-241 strong HIP phenotype expressing seeds treated with 5-Azacytidine showed a reduction in negative pleiotropic effects when rescued in vitro (Figure 8a), whereas we could not report a significant effect either on Lpa1/Lpa1 control (Figure 8b) or on Ipa1-1 individuals (data not shown). These results strongly suggest that the lpa1-241 allele could be silenced by means of epigenetic mechanisms involving DNA methylation. In line with this, lpa1-1 homozygotes were 5-Azacytidine insensitive, in accordance with the fact that a sequence mutation was found in the ZmMRP4 gene (Shi et al., 2007).

As previously reported for shoot tissue (Pilu et al., 2003), we confirmed that mips1S mRNA level is reduced in lpa1-241 mutants compared with wild type. In shoots obtained from 5-Azacytidine treated seeds, we observed a detectable increase in mips1s mRNA level (Figure 8c). A similar pattern was found for ZmMRP4 mRNA in the same tissues: its mRNA level seems reduced in lpa1-241 mutant compared with wild type, whereas 5-Azacytidine treatment causes a slight increase (Figure 8c). These results are consistent with the hypothesis that lpa1-241 mutant phenotype is due to epigenetic silencing of ZmMRP4 and, either directly or indirectly, mips1s gene. We may hypothesize that the reduced mips1s mRNA level independently found in developing seeds of lpa1-1 (Shukla et al., 2004) and in seeds and shoots of lpa1-241 mutants (Pilu et al., 2003), could be due to a metabolic feedback caused by an excess of myo-Ins phosphorylated intermediates or end products, such as phytic acid itself. In fact, MRP proteins in plants are vacuolar transporters involved in detoxification of both xenobiotics or endogenous substances (Klein et al., 2006), thus the loss of
ZmMRP4 activity may cause a cytosolic accumulation of its substrate, which may in turn feedback inhibit mips1s transcription. Another possibility is that the same epigenetic silencing phenomenon acting on ZmMRP4 gene may also silence mips1S gene.

Pleiotropic effects and phenotype reduction observed after 5-Azacytidine treatment can be primarily ascribed to an increase in ZmMRP4 expression level and transport activity, which, indirectly, may promote mips1s transcription by removing the hypothetical metabolic negative feedback. Alternatively, demethylation might remove epigenetic silencing marks from both ZmMRP4 and mips1s genomic sequences, restoring their transcription potential.

A further explanation may be that HIP phenotype and pleiotropic effects reduction in lpa1-241 mutant after 5-Azacytidine treatment may be because of an epigenetic activation of one or multiple genes, which contribute to partially overcome the lpa1-241 mutant phenotype.

Several mutants affecting paramutation have been isolated in maize: one mediator of paramutation1 (mop1) and three required to maintain repression ( $r m r 1, r m r 2$ and rmr6) (Dorweiler et al. 2000, Hollick and Chandler 2001, Hollick et al., 2005). Mop1 encodes for an RNAdependent RNA polymerase, which, together with Rmr2 (Hollick and Chandler 2001), is required for somatic maintenance of the paramutant state of $\mathrm{Pl}^{\prime}$ and $B^{\prime}$ but not $R^{\prime}$ allele. Mop1 is also required to establish silencing at $b 1$ and $r 1$ loci (Dorweiler et al. 2000, Alleman et al., 2006), and is able to progressively reactivate a silenced $M u D R$ element (Woodhouse et al., 2006).

Analysis of progenies obtained by crossing the mop1-1 mutant with our lpa mutants (Figure 6) showed that mop1-1 mutant is not involved in the maintenance of the silenced state of lpa1-241 allele. So far, we do not know whether paramutation is also affected, and we plan to do this experiment in the near future. Mop1 is an RNAdependent RNA polymerase possibly involved in maintaining a threshold level of some kind of silencing RNA, which mediates transcriptional gene silencing (Alleman et al., 2006). Although 5-Azacytidine can significantly revert lpa1-241 phenotype, mop1 mutation cannot. This evidence indicates that the hypothetical silencing phenomenon seems somewhat similar to $r 1$ locus paramutation (Brink, 1956).

Further studies will be necessary to understand the mechanism of establishment and maintenance of these lpa1 epialleles, and in particular we are planning to study chromatin structure in this region.

In conclusion, this is the first report of a paramutation phenomenon involving a fundamental metabolic pathway in maize, which might be the tip of an iceberg of homology-sensing mechanisms involved in several biological phenomena, not so far fully understood, such as heterosis.

\section{Acknowledgements}

This study was supported by Fondo Interno Ricerca Scientifica e Tecnologica (F.I.R.S.T. 2004, 2005 and 2006 to $\mathrm{R}$ Pilu). We thank Dr Victor Raboy, USDA ARS, Aberdeen, ID, USA, for his generous gift of lpa1-1 seeds, the anonymous referees for their precious comments and advices and Dr Davide Reginelli for his hard work in the field. 


\section{References}

Alleman M, Sidorenko L, McGinnis K, Seshadri V, Dorweiler JE, White J et al. (2006). An RNA-dependent RNA polymerase is required for paramutation in maize. Nature 442: 295-298.

Brink RA (1956). Change associated with the $R$ locus in maize is directed and potentially reversible. Genetics 41: 872-889.

Brown DF, Brink RA (1960). Paramutagenic action of paramutant $R^{r}$ and $R^{g}$ alleles in maize. Genetics 45: 1313-1316.

Chandler VL (2007). Paramutation: From maize to mice. Cell 128: 641-645.

Chandler VL, Eggleston WB, Dorweiler JE (2000). Paramutation in maize. Plant Mol Biol 43: 121-145.

Chandler VL, Stam M (2004). Chromatin conversations: mechanisms and implications of paramutation. Nat Rev Genet 5: 532-544.

Chen PS, Toribara TY, Warner H (1956). Microdetermination of phosphorus. Anal Chem 28: 1756-1758.

Coe Jr EH (1959). A regular and continuing conversion-type phenomenon at the $B$ locus in maize. Procs Natl Acad Sci USA 54: $828-832$

Coe Jr EH (1966). The properties, origin, and mechanism of conversion-type inheritance at the $B$ locus in maize. Genetics 53: 1035-1063.

Das OP, Messing J (1994). Variegated phenotype and developmental methylation changes of a maize allele originating from epimutation. Genetics 136: 1121-1141.

Della Vedova CB, Cone KC (2004). Paramutation: the chromatin connection. The Plant Cell 16: 1358-1364.

Dorweiler JE, Carey CC, Kubo KM, Hollick JB, Kermicle JL, Chandler VL (2000). mediator of paramutation1 is required for the establishment and maintenance of paramutation at multiple maize loci. The Plant Cell 12: 2101-2118.

Grant-Downton RT, Dickinson HG (2005). Epigenetics and its implications for plant biology. I. The epigenetic network in plants. Ann Bot 96: 1143-1164.

Hollick JB, Chandler VL (2001). Genetic factors required to maintain repression of a paramutagenic maize pl1 allele. Genetics 157: 369-378.

Hollick JB, Kermicle JL, Parkinson SE (2005). Rmr6 maintains meiotic inheritance of paramutant states in Zea mays. Genetics 171: 725-740.

Hollick JB, Patterson GI, Coe Jr EH, Cone KC, Chandler VL (1995). Allelic interactions heritably alter the activity of a metastable maize pl1 allele. Genetics 141: 709-719.

Johnson MD, Wang X (1996). Differentially expressed forms of 1L-myo-inositol 1-phosphate synthase (EC 5.51.4) in Phaseolus vulgaris. J Biol Chem 271: 17215-17218.

Klein M, Burla B, Martinoia E. (2006). The multidrug resistanceassociated protein (MRP/ABCC) subfamily of ATP-binding cassette transporters in plants. FEBS Lett 580: 1112-1122.

Lund G, Das OP, Messing J (1995). Tissue-specific dnase-Isensitive sites of the maize p-gene and their changes upon epimutation. Plant J 7: 797-807.

Majumder AL, Johnson MD, Henry SA (1997). 1L-myo-inositol 1-phosphate synthase. Biochem Biophys Acta 1348: 245-256.

Martienssen R (1996). Epigenetic phenomena: paramutation and gene silencing in plants. Curr Biol 6: 810-813.

Munnik T, Irvine RF, Musgrave A (1998). Phospholipid signalling in plants. Biochem Biophys Acta 1389: 222-272.

O'Dell BL, De Boland AR, Koirtyohann SR (1972). Distribution of phytate and nutritionally important elements among the morphological components of cereal grains. J Agric Food Chem 20: 718-721.
Pilu R, Landoni M, Cassani E, Doria E, Nielsen E (2005). The maize lpa241 mutation causes a remarkable variability of expression and some pleiotropic effects. Crop Sci 45: 2096-2105.

Pilu R, Panzeri D, Gavazzi G, Rasmussen S, Consonni G, Nielsen E (2003). Phenotypic, genetic and molecular characterization of a maize low phytic acid mutant (lpa241). Theor Appl Genet 107: 980-987.

Raboy V (1990). The biochemistry and genetic of phytic acid synthesis. In: Morre DJ, Boss W, Loewus FA (eds). Inositol Metabolism in Plants. Alan R Liss: New York, pp 52-73.

Raboy V, Gerbasi PF, Young KA, Stoneberg SD, Pickett SG, Bauman AT et al. (2000). Origin and seed phenotype of maize low phytic acid 1-1 and low phytic acid 2-1. Plant Physiol 124: 355-368.

Raychaudhuri A, Hait NC, DasGupta S, Bhaduri TJ, Deb R, Majumder AL (1997). L-myo-inositol 1-phosphate synthase from plant sources. Plant Physiol 115: 727-736.

Raychaudhuri A, Majumder AL (1996). Salinity-induced enhancement of L-myo-inositol 1-phosphate synthase in rice (Oryza sativa L.). Plant Cell Environ 19: 1437-1442.

Shi J, Wang H, Hazebroek J, Ertl DS, Harp T (2005). The maize low-phytic acid 3 encodes a myo-inositol kinase that plays a role in phytic acid biosynthesis in developing seeds. Plant $J$ 42: 708-719.

Shi JR, Wang HY, Schellin K, Li BL, Faller M, Stoop JM et al. (2007). Embryo-specific silencing of a transporter reduces phytic acid content of maize and soybean seeds. Nat Biotechnol 25: 930-937.

Shukla S, VanToai TT, Pratt RC (2004). Expression and nucleotide sequence of an INS (3) P1 synthase gene associated with low-phytate kernels in maize (Zea mays L.). J Agr Food Chem 52: 4565-4570.

Sidorenko LV, Peterson T (2001). Transgene-induced silencing identifies sequences involved in the establishment of paramutation of the Maize $p 1$ Gene. The Plant Cell 13: 319-335.

Stam M, Scheid OM (2005). Paramutation: an encounter leaving a lasting impression. Trends Plant Sci 10: 283-290.

Stevenson JM, Perera IY, Heilman I, Person S, Boss WF (2000). Inositol signaling and plant growth. Trends Plant Sci 5: 252-258.

Swarbreck D, Ripoll PJ, Brown DA, Edwards KJ, Theodoulou F (2003). Isolation and characterisation of two multidrug resistance associated protein genes from maize. Gene 315: 153-164.

van Tunen AJ, Koes RE, Spelt CE, van der Kroll AR, Stuitje AR, Mol JNM (1988). Cloning of two chalcone flavanone isomerase genes from Petunia hybrida: coordinate, light regulated and differential expression of flavonoid genes. EMBO J 14: 2350-2363.

Vaucheret H (2006). Post-transcriptional small RNA pathways in plants: mechanisms and regulations. Genes Dev 20: 759-771.

Wolffe AP, Matzke MA (1999). Epigenetics: regulation through repression. Science 286: 481-486.

Woodhouse MR, Freeling M, Lisch D (2006). The mop1 (mediator of paramutation1) utant progressively reactivates one of the two genes encoded by the $M u D R$ transposon in maize. Genetics 172: 579-592.

Wright AD, Moehlenkamp CA, Perrot GH, Neuffer MG, Cone KC (1992). The maize auxtrophic mutant orange pericarp is defective in duplicate genes for tryptophan synthase. The Plant Cell 4: 711-719. 